\title{
Prevención del riesgo de lavado de activos. Caso Colombia y Ecuador
}

\author{
Prevention of the risk of money laundering. \\ Case Colombia and Ecuador \\ María Tamara Ortiz Luzuriaga \\ José Flores Sanchez \\ Walter Caicedo Leones \\ Marjorie Ordoñez Guartazaca
}




\title{
Prevención del riesgo de lavado de activos. Caso Colombia y Ecuador
}

\author{
Prevention of the risk of money laundering. Case Colombia and Ecuador \\ María Tamara Ortiz Luzuriaga ${ }^{1}$. Jose Flores Sanchez ${ }^{2}$. Walter Caicedo Leones ${ }^{3}$.Marjorie Ordonez \\ Guartazaca $^{4}$ \\ Como citar: Ortiz. M., Flores. J., Caicedo. W., Ordoñez M., (2021). Prevención del riesgo de lavado de activos. Caso Colombia y Ecuador. Revista Universidad de \\ Guayaquil. 133(2).27-40 doi: https://doi.org/10.53591/rug.v133i2.1391
}

\section{RESUMEN}

El lavado de activos es un problema que afecta a la economía de los países, pues genera una emisión monetaria que fortalece mercados ilícitos extra bancarios con procedimientos cada vez más complejos de operación, y cuyas actividades son de alcance internacional. El Ecuador presenta altos niveles de riesgo a actividades ilícitas como contrabando de dinero o lavado de activos debido a que se encuentra en una zona geográfica con altos niveles de corrupción. Consecuentemente, este artículo tiene como propósito resumir un marco referencial de las principales metodologías para la detección del riesgo de este fenómeno que afecta a la sociedad. Para esto, se siguió una metodología con enfoque cualitativo basada en la revisión documental de base de datos científicas como también en reportes anuales de organismos de control. Se evidencia que la regulación colombiana considera varios modelos dirigidos a diferentes actividades económica para identificar, prevenir y monitorear el riesgo.

Palabras Clave: Lavado de activos. Mercados Ilícitos, Detección del Riesgo.

\section{ABSTRACT}

The money laundering is a problem that affects the economies of the countries, since it generates a monetary issue that strengthens illicit extra-bank markets with increasingly complex operating procedures, and whose activities are international in scope. Ecuador presents high levels of risk to illicit activities such as money smuggling or money laundering because it is in a geographical area with high levels of corruption. Consequently, this article aims to summarize a referential framework of the main methodologies for detecting the risk of this phenomenon that affects society. For this, a methodology was followed with a qualitative approach based on the documentary review of scientific databases as well as annual reports from control bodies. It is evident that Colombian regulation considers several models aimed at different economic activities to identify, prevent and monitor risk

Keywords: Money laundering. Illicit Markets, Risk Detection.

\footnotetext{
${ }^{1}$ Magister en Administración de Empresas, Docente de la Facultad de Ciencias Administrativas, Universidad de Guayaquil, Ecuador. Correo electrónico: maria.ortizl@ug.edu.ec.

${ }^{2}$ Magister en Administración de Empresas, Docente de la Facultad de Ciencias Administrativas, Universidad de Guayaquil, Ecuador. Correo electrónico: jose.floressa@ug.edu.ec

${ }^{3}$ Negocios Internacionales y Gestión de comercio exterior, Docente de la Facultad de Ciencias Administrativas, Universidad de Guayaquil, Ecuador. Correo electrónico: walter.caicedol@ug.edu.ec.

${ }^{4}$ Magister en Administración de Empresas, Docente de la Facultad de Ciencias Administrativas, Universidad de Guayaquil, Ecuador. Correo electrónico: marjorie.ordonezg@ug.edu.ec
} 


\section{INTRODUCCIÓN}

En un mundo globalizado algunos bancos a través de sus filiales ubicadas en paraísos fiscales descubren nuevas posibilidades de negocio para el manejo de los dólares de los narcotraficantes, pues no cobran intereses o rendimientos por sus depósitos, a cambio de la protección de fondos mediante el secreto bancario. Las instituciones bancarias con el avance de los años han crecido en tamaño, de actividades operacionales y servicios financieros que permiten al cliente tomar decisiones frente a un mercado financiero diverso de ofertas para incentivar el ahorro y la inversión.

Sin embargo, las categorías de crédito establecidas no permiten reflejar correctamente el destino del crédito de las entidades financieras, reflejándose en las estadísticas como créditos comerciales, pero que en realidad se destinan al fomento de la producción agropecuaria, pequeña industria, turismo, servicios, comercio y construcción de vivienda y de infraestructura en general (Salgado, 2010, pág. 61). En consecuencia, se dificulta el análisis del destino de los créditos por actividades productivas se dificulta desde la perspectiva de análisis de riesgo de lavado de activos y posibles delitos asociados.

Cabe mencionar, que el lavado de activos en el Ecuador comienza a tener mayor impacto a partir de la dolarización de la economía (2000), cuando se produjo una reconfiguración del narcotráfico a escala global, el país de tránsito de drogas y otros bienes de procedencia ilegal, pasó también a ser país de "lavado de activos". Se considera lavado de activos a los actos que dan apariencia de legalidad a patrimonios provenientes de actividades ilegales. Por lo tanto, el objetivo de estas operaciones es utilizar a la empresa como instrumento para el ocultamiento, manejo, inversión o aprovechamiento de cualquier forma de dinero o cualquier otra clase de bienes, tales como inmuebles, vehículos, semovientes, bonos, certificados de depósito a término y otros títulos transables en el mercado bursátil, entre otros (Zabala Leal, 2019, pág. 9).

Para efectos de la normativa de prevención de lavado de activos, financiamiento del terrorismo y otros delitos, se define a los activos como:

los bienes; los activos financieros; las propiedades de toda clase, tangibles o intangibles; los muebles o inmuebles, con independencia de cómo se hubieran obtenido; y los documentos o instrumentos legales, sea cual fuere su forma, incluida la electrónica o digital, que acrediten la propiedad u otros derechos sobre dichos bienes, sean estos, entre otros, créditos bancarios, cheques bancarios o de viajero, acciones, títulos, obligaciones, letras de cambio, cartas de crédito, y los intereses, dividendos u otros ingresos o valores que se devenguen o sean generados por esos fondos u otros bienes (SCVS, 2019, pág. 2).

A criterio de Arias (2015) las entidades gubernamentales de control del Ecuador han realizado análisis donde determinan una alta actividad en conversión o transferencia de capitales de origen ilícito de un país a otro insertándolo en el sistema económico, por tal razón creó la Ley de prevención, detección y erradicación del delito de lavado de activo que antecede en el modelo del Sistema Unitario De Compensación Regional. Los principales objetivos de la ley fueron disminuir el uso del dólar y otras divisas internacionales entre los miembros del ALBA, que es una organización internacional formada por países de América Latina y el Caribe donde firmaron tratados de la lucha contra la pobreza y la exclusión social con bases socialistas, con el fin de minimizar el impacto económico y la vulnerabilidad del uso de otras monedas en sus economías. 
En cambie, en sus diferentes modalidades y tiene por objeto reprimir la propiedad, posesión, utilización, oferta, venta, corretaje, comercio interno o externo, transferencia, conversión y tráfico de activos, que fueren resultado o producto de actividades ilícitas, o constituyan instrumentos de ellas y la financiación de delitos, en sus diferentes modalidades detectando operaciones inusuales en sus actividades y transacciones económicas (Arias, 2015).

El estudio realizado por el GAFISUD (2011), Grupo de Acción Financiera de Sudamérica, evaluó el marco institucional, sistemas regulatorios y otros sistemas vigentes para dar cumplimiento de las leyes, regulaciones y materiales provistos para el control de lavado de activos en el Ecuador, y con esto proponer estrategias de control para la disuasión de actividades delictivas. El resultado de la evaluación fue aprobado el 15 de diciembre de 2011 y estableció un proceso de "Seguimiento Intensificado" debido a que Recomendaciones claves del GAFI se encontraron calificadas con un parcialmente cumplido.

Frente a los resultados hallados, el GAFISUD (2011) recomendó que las entidades públicas y privadas ejecuten el programa y actividades diseñadas por la Unidad de Análisis Financiero para la prevención del riesgo y así alcanzar los objetivos de la ley. Esto significa que la autoridad competente atenderá pedidos de tribunales o autoridades similares de otros Estados, para la prestación de asistencia recíproca en relación con investigaciones o procedimientos de carácter administrativo, civil o penal, que tengan relación con el lavado de activos y el financiamiento de delitos. Finalmente, en el 2015 el Ecuador fue excluido de la clasificación del GAFI como jurisdicción con deficiencias estratégica. Disposición que se logró mediante el cumplimiento sustancial -a nivel técnico-, de todas las actividades previstas en el Plan de Acción Continuo del país y, principalmente, la existencia de un marco legal e institucional satisfactorio que rige al sistema nacional antilavado de activos y contra el financiamiento del terrorismo (ALA/CFT) (PGE, 2015).

Actualmente, Ecuador cuenta con el Plan Nacional de Prevención Integral y Control del Fenómeno Socio Económico de Drogas 2017-2021, en el cual se considera fortalecer, los marcos normativos e institucionales para contrarrestar el lavado de activos derivado del tráfico ilícito de drogas. El análisis del riesgos y reportes evidencian que la ubicación geográfica del Ecuador facilita la operación de actividades ilícitas pues se encuentra en medio de los dos grandes productores de narcóticos, presentándose la necesidad de actualizar el marco legal y adoptar metodologías de evaluación de riesgo, prevención y control de lavado de activos aplicados a los diversos sectores de la economía.

\section{El riesgo de lavado de activos y financiamiento del terrorismo}

El riesgo de lavado de activos y financiamiento de delitos como el terrorismo se considera a la posibilidad de pérdida o daño que puede sufrir una entidad controlada por su exposición a ser utilizada directamente o a través de sus operaciones como instrumento para el lavado de activos y/o canalización de recursos hacia la realización de actividades delictivas incluida el terrorismo, o cuando se pretenda el ocultamiento de activos provenientes de dichas actividades. El riesgo de lavado de activos y financiamiento de otros delitos se materializa a través de los riesgos asociados: legal, reputacional (desprestigio o mala imagen), operativo (fallas en los procesos, recursos, tecnología e infraestructura) y de contagio por las acciones de una persona vinculada a la empresa (Superintencia de Bancos, 2020). Estos riesgos son consideraciones adicionales al análisis de la gestión estratégica 


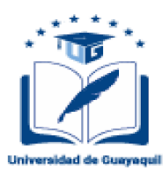

del negocio, en la cual cada actividad económica posee características inherentes al giro de sus operaciones.

El riesgo se puede definir como la posibilidad de que ocurra un evento que tendrá un impacto sobre los objetivos, o que ocurran afectaciones sobre los objetivos esperados, se mide en términos de consecuencias y probabilidades. La Norma de Gestión de Riesgos ISO 31000: Estándar de Australia y Nueva Zelanda sobre administración de riesgos: AS/NZS: 4360, identifica varios tipos de riesgos: reputacional, legal, operativo, de contrato e inherente.

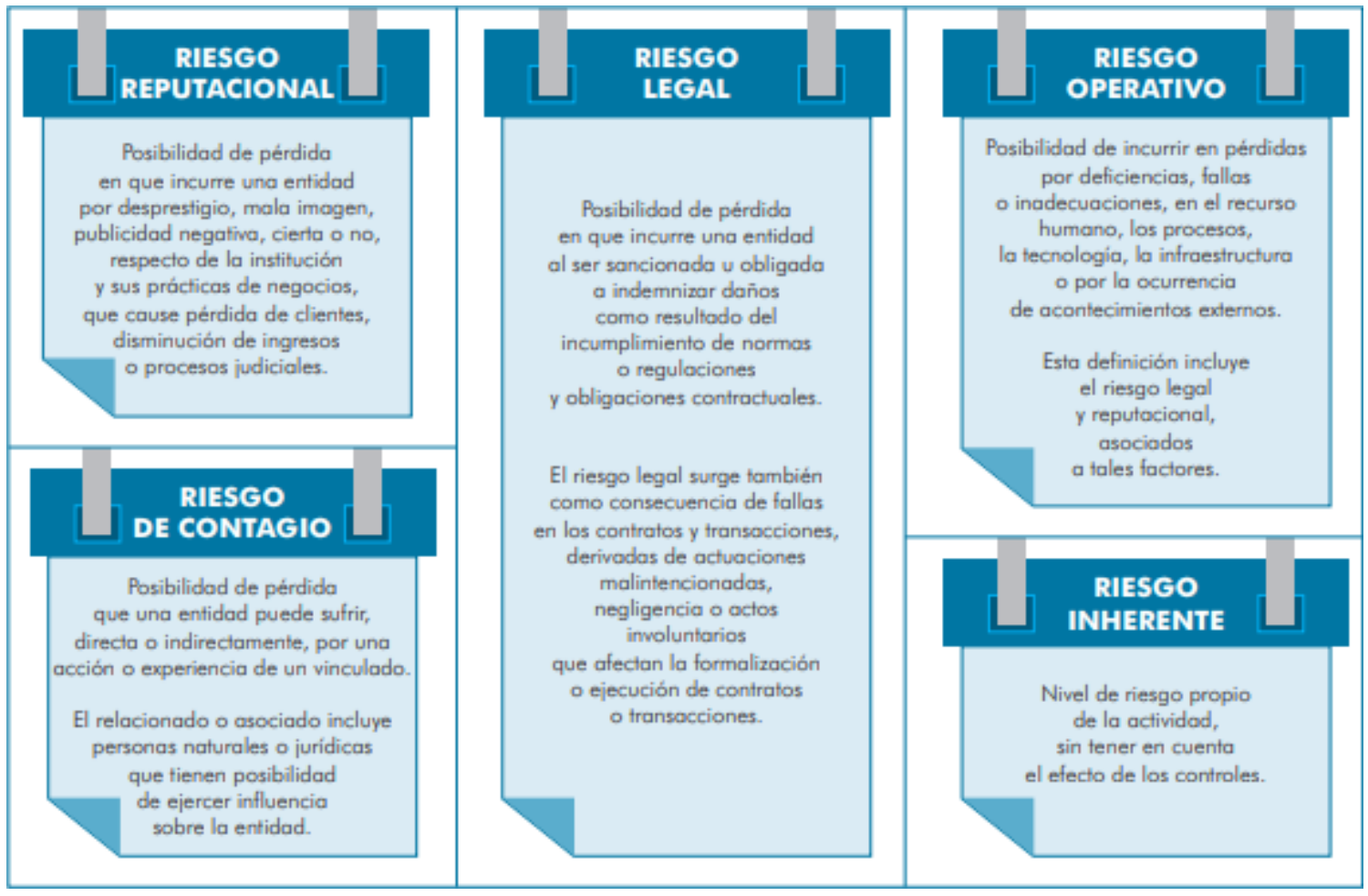

Figura 1. Tipos de riesgos

Tomado de: Guía anticorrupción para las empresas. Basado en el Estatuto Anticorrupción (UNODC, 2017, pág. 36)

Los riesgos asociados a la corrupción poseen consecuencias tangibles e inclusive pueden afectar la sostenibilidad de las operaciones y continuidad del negocio. En el Ecuador, en el año 2020, la Unidad de Análisis Financiero y Económico UAFE estableció una guía para que los sujetos obligados puedan tener un mejor entendimiento sobre el alcance de la definición de PEP y los criterios para su designación con enfoque basado en riesgo. El mencionado documento se realizó teniendo en consideración la recomendación 40 del GAFI. En este sentido, Colombia emitió un documento similar con la participación de las Naciones Unidas: Guía anticorrupción para las empresas. Basado en el Estatuto Anticorrupción.

En la Tabla 1 se presenta la definición comparativa para lo tipos de riesgos relacionadas con las actividades de lavado de activos. 
Tipos de Riesgos

\begin{tabular}{|c|c|c|}
\hline Riesgo & $\begin{array}{c}\text { Cámara de Comercio de } \\
\text { Colombia }\end{array}$ & $\begin{array}{c}\text { Unidad de Análisis Financiero y } \\
\text { Económico }\end{array}$ \\
\hline Legal & $\begin{array}{l}\text { Procesos penales, Sanciones, } \\
\text { Demandas civiles, Multas. }\end{array}$ & $\begin{array}{l}\text { Posibilidad de pérdida por sanción, multa o } \\
\text { indemnización por incumplimiento de Leyes, } \\
\text { Normas o Instructivos. }\end{array}$ \\
\hline Reputacional & $\begin{array}{l}\text { Escándalo en medios de } \\
\text { comunicación. Publicaciones } \\
\text { negativas en redes sociales. }\end{array}$ & $\begin{array}{l}\text { Probabilidad de pérdida por mala imagen, } \\
\text { desprestigio de la empresa o sus negocios. }\end{array}$ \\
\hline Operativo & $\begin{array}{l}\text { Cierre temporal de las empresas } \\
\text { Embargos por procesos penales } \\
\text { Ocupaciones con fines de } \\
\text { extinción de dominio. }\end{array}$ & $\begin{array}{l}\text { Riesgo de pérdida por deficiencia o fallas en } \\
\text { el recurso humano, procesos, tecnología y } \\
\text { acontecimientos externos. }\end{array}$ \\
\hline Contagio & $\begin{array}{l}\text { Extensión de escándalo a } \\
\text { empresas del grupo Afectación de } \\
\text { bienes a empresas del grupo. }\end{array}$ & $\begin{array}{l}\text { Probabilidad de pérdida por acción o } \\
\text { experiencia de un relacionado o personas que } \\
\text { pueden ejercer influencia. }\end{array}$ \\
\hline Inherente & $\begin{array}{l}\text { Modificación de reglas de juego en } \\
\text { el sector o actividad económica } \\
\text { Señalamiento del sector o de la } \\
\text { actividad económica. }\end{array}$ & $\begin{array}{l}\text { Es el riesgo intrínseco de cada factor de riesgo } \\
\text { (producto, servicio, cliente, etc.), derivado de } \\
\text { su propia naturaleza o características; éste } \\
\text { surge de la exposición y la incertidumbre de } \\
\text { probables eventos. Puede causar un impacto } \\
\text { financiero en el sujeto obligado. }\end{array}$ \\
\hline
\end{tabular}

\section{Estadísticas operaciones inusuales e injustificadas en el Ecuador}

La Unidad de Análisis Financiero y Económico, colabora con la Fiscalía General del Estado (FGE) y los órganos jurisdiccionales proporcionando la información necesaria para la investigación, procesamiento y juzgamiento de los delitos de lavado de activos y financiamiento de delitos. En este sentido la Dirección de Análisis de Operaciones atendió 811 peticiones provenientes de la Fiscalía General del Estado en el año 2019; esto significó un incremento del 3\% frente a las 787 respuestas enviadas en el año 2018. Es importante recalcar que los requerimientos con mayor demanda se relacionan con el lavado de activos, representado en el 19\% de los casos del año 2019 (UAFE, 2020).

La Dirección de Análisis de Operaciones de la UAFE remitió a la FGE, reportes de operaciones inusuales e injustificadas (ROII), los cuales, en el informe de la rendición de cuentas se expresan bajo diferentes tipologías para los años 2018 y 2019, dificultando el análisis comparativo de la información. Para la normativa ecuatoriana las operaciones o transacciones económicas inusuales, injustificadas o sospechosas son:

los movimientos económicos, realizados por personas naturales o jurídicas, que no guarden correspondencia con el perfil económico y financiero que estas han mantenido con el sujeto obligado y que no puedan sustentarse (SCVS, 2019, pág. 4).

En las Tabla 2 y Tabla 3 se detallan las tipologías determinadas en cada reporte, así como el monto 
involucrado.

Tabla 2.

Operaciones inusuales e injustificadas reportadas en Ecuador en el año 2018

\begin{tabular}{|c|c|c|c|c|}
\hline \multirow{2}{*}{ Tipologías } & \multirow{2}{*}{$\begin{array}{l}\text { No. } \\
\text { Casos }\end{array}$} & \multicolumn{2}{|c|}{$\begin{array}{c}\text { No de Personas } \\
\text { Relacionadas }\end{array}$} & \multirow{2}{*}{$\begin{array}{r}\text { Monto } \\
\text { Involucrado }\end{array}$} \\
\hline & & Naturales & Jurídicas & \\
\hline $\begin{array}{l}\text { Adquisición de bienes no acorde al perfil } \\
\text { económico }\end{array}$ & 1 & 2 & & $494.247,33$ \\
\hline $\begin{array}{l}\text { Aumento de capital en aseguradora que no cuentan } \\
\text { con justificación }\end{array}$ & 1 & 2 & 1 & $172.536 .145,72$ \\
\hline $\begin{array}{l}\text { Exportación de oro de origen ilícito utilizando } \\
\text { empresaslegítimas }\end{array}$ & 2 & 5 & 13 & $330.102 .385,37$ \\
\hline $\begin{array}{l}\text { Ingreso de bienes y capitales derivados de actos de } \\
\text { corrupción, PEP }\end{array}$ & 13 & 26 & 7 & $215.847 .485,76$ \\
\hline $\begin{array}{l}\text { Lavado de dinero producto de actividades } \\
\text { relacionadasal narcotráfico }\end{array}$ & 1 & 2 & 1 & $1.048 .349,00$ \\
\hline Uso de fideicomisos para ocultar bienes ilícitos & 1 & 5 & 2 & 25.521.913,13 \\
\hline TOTAL & 19 & 42 & 24 & $745.550 .526,31$ \\
\hline
\end{tabular}

Tomado de: Informe de Gestión Anual Ejercicio 2018 (Salazar Méndez, 2019).

Tabla 3.

Operaciones inusuales e injustificadas reportadas en Ecuador en el año 2019

\begin{tabular}{|c|c|c|c|c|}
\hline \multirow[t]{2}{*}{ Tipologías } & \multirow{2}{*}{$\begin{array}{l}\text { No. } \\
\text { Casos }\end{array}$} & \multicolumn{2}{|c|}{$\begin{array}{l}\text { No de Personas } \\
\text { Relacionadas por Tipo }\end{array}$} & \multirow{2}{*}{$\begin{array}{c}\text { Monto } \\
\text { Involucrado }\end{array}$} \\
\hline & & Natural & Jurídica & \\
\hline Aumento de patrimonio sin justificación & 2 & 2 & & $4.193 .007,36$ \\
\hline Corrupción pública & 6 & 9 & 5 & $21.854 .388,43$ \\
\hline Encubrimiento tras estructuras comerciales & 1 & 1 & & $3.234 .754,87$ \\
\hline $\begin{array}{l}\text { Mal uso de negocios legítimos / uso de } \\
\text { Testaferros }\end{array}$ & 1 & 1 & & 4.647.130,45 \\
\hline Mal uso de productos y servicios bancarios & 3 & 7 & 2 & 12.087.704,61 \\
\hline $\begin{array}{l}\text { Obtención de bienes y capitales no acordes } \\
\text { al perfil económico }\end{array}$ & 4 & 9 & & 29.179.113,24 \\
\hline $\begin{array}{l}\text { Uso de identidades falsas, documentos o } \\
\text { Testaferros }\end{array}$ & 1 & 1 & 4 & $18.388 .622,40$ \\
\hline Utilización de compañías fachada & 1 & 1 & 1 & $5.016 .468,55$ \\
\hline TOTAL & 19 & 31 & 12 & 98.601.189,91 \\
\hline
\end{tabular}

Tomado de: Informe de Gestión 2019 Unidad de Análisis Financiero y Económico (UAFE, 2020)

En el año 2020 las respuestas emitidas por la UAFE a peticiones de Fiscalía General del Estado han sido motivadas con mayor frecuencia por el delito de peculado, siendo el 17\% de los requerimientos; seguidos de lavado de activo en un $11 \%$ y tráfico ilícito de sustancias catalogadas sujetas a Fiscalización en un $10,8 \%$. 
Tabla 4.

Requerimientos de información FGE en el año 2020

\begin{tabular}{lrc}
\hline \multicolumn{1}{c}{ ELITO } & TOTAL & PARTICIPACION \\
\hline Peculado & 163 & $17,0 \%$ \\
Lavado de activos & 105 & $11,0 \%$ \\
Tráfico ilícito de sustancias catalogadas sujetas a Fiscalización & 103 & $10,8 \%$ \\
Falsificación y uso de documento falso & 72 & $7,5 \%$ \\
Defraudación tributaria & 69 & $7,2 \%$ \\
Investigación & 64 & $6,7 \%$ \\
Asociación ilícita & 49 & $5,1 \%$ \\
Enriquecimiento privado no justificado & 38 & $4,0 \%$ \\
Estafa & 36 & $3,8 \%$ \\
Enriquecimiento ilícito & 35 & $3,7 \%$ \\
Delincuencia organizada & 34 & $3,5 \%$ \\
Tráfico de influencias & 30 & $3,1 \%$ \\
Concusión & 21 & $2,2 \%$ \\
Abuso de confianza & 19 & $2,0 \%$ \\
Otros & 69 & $7,2 \%$ \\
\hline TOTAL & 958 & $100 \%$ \\
\hline
\end{tabular}

Adaptado de: Informe de Gestión Enero-Diciembre 2020.Unidad de Análisis Financiero y Económico (UAFE, 2021)

Así que, es notable los resultados del crecimiento de lavado de activos y operaciones inusuales en el Ecuador, mismos que han afectado la sociedad, la seguridad, los sectores económicos, la educación frente a un mercado ilícito de narcotráfico, trata de blancas, distribución de droga que compra conciencias de personas vulnerables con escasos recursos para que sean partícipes de las redes del narcotráfico que fomentan mediante acciones ilícitas, la violación de los derechos constitucionales del país y el bienestar de la sociedad.

Por lo tanto, es importante identificar y antelarse ante todos los eventos previniendo situaciones que afecten la economía social del país, aplicando modelos eficientes de lavado de activos que permitan diagnosticar los comportamientos de los eventos, mediante un seguimiento y control de la normas y leyes que permitan con efectividad y transparencia resolver los problemas antes de que comiencen a crecer, para salvaguardar la vida, la salud, el bienestar, seguridad, la economía popular y solidaria de las millones de familias ecuatorianas.

Para Guerrero et al (2019), el estado ecuatoriano debe incrementar datos estadísticos e investigaciones en materia del lavado de activos, que sean de fácil acceso que permitan análisis cuantitativos con el fin de realizar la medición y afectación de las actividades ilícitas, es decir, conocer el impacto real en la economía del país desde el ámbito productivo y económico. Con este fin, en el mes de Agosto del 2020, se ha creado la Unidad de Análisis Estratégico para realizar estudios para la identificación de prácticas, mecanismos, técnicas y tipologías empleadas en el lavado de activos y el financiamiento 
de delitos en los diferentes sectores económicos. Además, mediante la resolución UAFE-DG-20200089 NORMAS PLA-FT, se incrementó los sectores controlados, tales como: Registro Mercantil, ONGs, Notarias y Registro de la propiedad.

\section{Metodología para el control del lavado de activos}

El lavado de dinero es un delito que permite blanquear capitales que fueron adquiridos en forma ilícita, que comúnmente se llevan a cabo en países donde sus leyes muestran falencias para sancionar este delito. Según Carvini (2002) este capital es invertido, sustituido o transformado en algún tipo de negocio, incorporándolo en los circuitos económicos, financieros y legales. Anteriormente, al lavado de capitales se lo refería como blanqueo por ser considerarse un proceso de lavar una y otra vez ese capital sucio, considerado como negro, hasta que la riqueza no tenga manchas (trazabilidad) de su procedencia ilegal, es decir, que no se pueda identificar su verdadera naturaleza.

Colombia es el país que se encuentra a la mira global por ser el país productor de plantaciones de coca más grande, y donde se distribuye la droga al mundo, estableciendo redes entre grupos de poder para influir en el acceso libre de la droga al exterior, financiando guerrillas que protegen plantaciones para velar por los intereses de los carteles que compran droga desde la región centro andina.

El Sistema Integral para la Prevención y Control del Lavado de Activos y Financiación del Terrorismo - SIPLAFT y el Sistema de Administración de Riesgo de Lavado de Activos y Financiamiento del Terrorismo (SARLAFT), son metodologías colombianas que permiten identificar y monitorear de manera eficiente estas actividades delictivas. En Colombia el sistema SARLAFT se aplica a varios sectores regulados por diferentes entes de control, tales (UIAF, 2019):

- Superintendencia Financiera de Colombia - SFC: fondos de garantías, sociedades de calificadoras de valores y/riesgos, intermediarios de reaseguros, etc.

- Superintendencia de Sociedades - Sociedades Comerciales: inmobiliarias, construcción de edificios, explotación de minas y canteras, servicios jurídicos, servicios contables, de cobranza y/o de calificación crediticia, e comercio de vehículos, sus partes, piezas y accesorios

- Superintendencia de Vigilancia y Seguridad Privada: Empresas y cooperativas de vigilancia y seguridad privada, escuelas de capacitación y entrenamiento, Servicios de asesoría, consultoría e investigación de seguridad, fabricación, instalación, comercialización y utilización de equipos para la vigilancia y la seguridad privada.

- Superintendencia Nacional de Salud: Agentes del Sistema General de Seguridad Social en Salud, Entidades Promotoras de Salud (EPS) del régimen contributivo y subsidiado, Instituciones Prestadoras de Salud (IPS) públicas y privadas, Empresas de Medicina Prepagada, Entidades que prestan servicios de Ambulancia Prepagada, Empresas Promotoras de Salud Indígenas.

- Ministerio de las Tecnologías de la Información y Las Comunicaciones - MINTIC: operadores de servicios postales

La Dirección de Impuestos y Aduanas Nacionales DIAN del estado colombiano, en el año 2002 estableció la adopción del sistema SIPLA, que determinar elaborar un manual de procedimientos orientados al conocimiento del cliente y del mercado, funciones y categoría del empleado de 
cumplimiento, sanciones y correctivos. Posteriormente, en el año 2016, la Superintendencia de Puertos y Transporte señaló los criterios técnicos y jurídicos que deben cumplir las empresas de transporte terrestre de carga sujetas a su vigilancia, inspección y control, con el objetivo que se implemente un sistema integral para la prevención y control del lavado de activos, la financiación del terrorismo y el financiamiento de la proliferación de armas de destrucción (SIPLAFT) (Infolaft, s.f.).

Para ampliar el alcance de la prevención de lavado de activos, la recomendación 28 del GAFI menciona que es necesario establecer medidas sobre Actividades y Profesiones No Financieras Designadas APNFD. Por lo tanto, Colombia implementó la metodología SAGRILAFT, que es la sigla de Sistema de Autocontrol y Gestión del Riesgo Integral de Lavado de Activos y Financiación del Terrorismo. El ámbito de aplicación es para empresas vigiladas por la Superintendencia de Sociedades pertenecientes a diferentes sectores como: el inmobiliario, servicios jurídicos, contables, de cobranza, comercio de vehículos y construcción de edificios (Infolaft).

Otros sistemas de Antilavado de Activos y Contra la Financiación del Terrorismo - ALA/CFT en Colombia son:

- Sector vigilado por el Ministerio de Tecnologías de la Información y las Comunicaciones MINTIC: Sistema de Administración del Riesgo de Lavado de Activos y de la Financiación del Terrorismo y la Financiación de la Proliferación de Armas de Destrucción Masiva SARLAFT- FPADM.

- Sector vigilado por la Junta Central de Contadores - Revisores Fiscales: Sistema de Autocontrol y Gestión del Riesgo de Lavado de Activos y Financiación del Terrorismo SAGRLAFT

\section{Metodología de implementación SARLAFT}

La legislación colombiana ha desarrollado importantes avances en la prevención y control de lavado de activos, para lo cual, estableció el Sistema de Administración del Riesgo de Lavado de Activos y Financiación del Terrorismo, SARLAFT. Esta normativa se desarrolló en concordancia con las definiciones y/o recomendaciones del GAFI, e incluye la adopción de 8 elementos: políticas, procedimientos documentación, estructura organizacional, órganos de control, infraestructura tecnológica, divulgación y capacitación (Superintendencia de la Economía Solidaria, 2017, pág. 21).

El modelo permite analizar la capacidad del instrumento y la eficacia de los controles del sistema en la efectividad del cumplimiento, con el objetivo de que las entidades vigiladas implementen SARLAFT a fin de prevenir que las mismas sean utilizadas para dar apariencia de legalidad a activos provenientes de actividades delictivas o para la canalización de recursos hacia la realización de actividades terroristas (Saá, 2013)

La metodología del Sistema para la Administración del Riesgo de Lavado de Activos y de la Financiación del Terrorismo (SARLAFT), consiste en disminuir la probabilidad o gravedad de pérdida, cumpliendo con las normas y gestionando la dificultad de identificar, evaluar, medir, mitigar y monitorear, para lo cual hay que tener muy presente: los estándares de gestión del riesgo, control interno, los índices de medición del riesgo del Comité de Basilea II y el Estándar Australiano de Administración del Riesgo AS/NZS 4360 que utiliza como guía el Modelo establecido en la Norma 


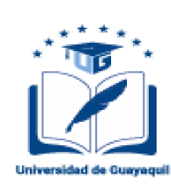

Técnica Colombiana NTC 5254 Gestión del Riesgo, que permite establecer e implementar el proceso de administración del riesgo de lavado de activos y financiamiento del terrorismo en una entidad bancaria (ICONTEC, 2004).

La contextualización del riesgo permite a la organización en la implementación de la metodología SARLAFT evaluar y entender el entorno externo e interno, el ámbito social, cultural y político, e inclusive identificar cuáles son las circunstancias del país y los procesos de observación o evaluación internacional. En la evaluación del contexto externo se debe terne en cuenta varios aspectos, tales como: (Superintendencia de la Economía Solidaria, 2017, pág. 28):

- El ambiente social, cultural y político

- El marco legal y/o reglamentario

- El ámbito financiero, tecnológico, natural y competitivo y,

- Contexto nacional regional y local en la gestión del riesgo.

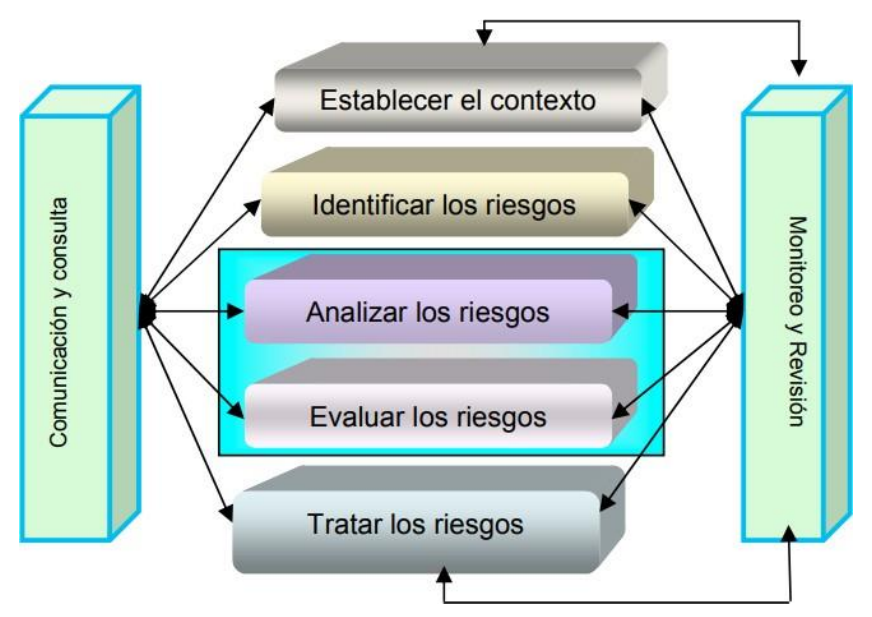

Figura 2. Modelo de administración del riesgo.

La metodología de SARLAFT está dispuesta por la Superintendencia de Bancos de Colombia, básicamente abarca lo siguiente: como políticas, aplicación, estructura organizacional de un SARLAFT, funciones y responsabilidades de los participantes en la Administración del Riesgo de LA/FT, metodologías para la segmentación de clientes, proveedores y empleados, tipologías, medidas que aseguran el cumplimiento de las políticas del SARLAFT, procedimientos y Metodologías para identificar, medir, controlar y monitorear el riesgo de LA/FT, procedimientos de los órganos de control frente al SARLAFT, Programas de Capacitación, Control de Cambios y Anexos (SARLAFT, 2012). No obstante, el modelo presenta en su estructuración seis fases las cuales determina las etapas de construcción del modelo de la siguiente manera:

Etapa 1: Etapa de determinación del marco conceptual y el contexto externo e interno de la entidad. Etapa 2: Etapa de identificación de los riesgos de la entidad.

Etapa 3: Etapa de evaluación y medición de los riesgos.

Etapa 4: Etapa de Control y Detección.

Etapa 5: Monitoreo.

Etapa 6: Comunicación, Consulta y Capacitación. Reportes. 
Se mostraron de manera descriptiva los componentes que inciden en la estructuración del modelo SARFLAT, mismo que permite a las entidades un mayor control y seguimiento de los mecanismos de control en el lavado de activos con el fin de salvaguardar y garantizar la seguridad de la nación frente a acciones delictivas de grupos que quieren alterar el orden social de una nación por intereses, violentando contra toda ley jurídica que se encuentra por encima de ellos.

La metodología SARLAFT ha sido validada por académicos en la literatura como Mariño, Chaparro y Medina (2014), que se preocuparon por hallar los determinantes en la prevención del riesgo de estas operaciones delictivas. Realizaron una investigación con enfoque cualitativo y de alcance descriptivo analizando casos en que ha sido implementado el sistema SARLAFT en la administración del sector público y privado. Como herramienta de medición de riesgo utilizaron una matriz de factor de riesgo de personas elaborado para la empresa objeto de estudio realizado por etapas. Finalmente, como resultado de la investigación plantearon que son necesarias tres herramientas de orden administrativo: la matriz de identificación y cuantificación del riesgo, manual de procedimientos de prevención del riesgo para lavado de activos, y sistema de información (Mariño Becerra, Chaparro Gómez, \& Medina Sandoval, 2014)

\section{CONCLUSIONES}

Las compañías colombianas mediante la aplicación de las metodologías SIPLAFT, SAGRLAFT y SARLAFT establecen lineamientos que precisen la conducta esperada de los colaboradores frente a situaciones como conflictos de interés, y manejo de información mediante la utilización de un manual para la prevención y control del lavado de activos y de financiación del terrorismo. Se busca poner en práctica procedimientos propios, dando cumplimiento al diseño e implementación de acuerdo con las buenas prácticas, generando dentro de la organización una cultura orientada al cumplimiento de las normas establecidas en materia.

En países como Ecuador, existe escasa información pública de fácil acceso para una mejor evaluación y monitoreo del riesgo del lavado de activos y financiamiento del terrorismo. Además, existe poca evidencia estadística para proyectar resultados y predecir un estado de peligro. En consecuencia, se requiere realizar estudios que faciliten determinar factores, criterios y categorías de riesgo de prevención de lavado de activos, financiamiento del terrorismo y otros delitos asociados; y el análisis del mercado, perfil operacional y comportamiento de accionistas, empleados, clientes y proveedores.

Por otro lado, la literatura demuestra constante interés en el estudio del fenómeno del lavado de dinero y financiamiento del terrorismo, no solo de académicos sino también de profesionales en auditoría forense que intentan estandarizar un modelo que ayude a prevenir el riesgo de estas actividades ilícitas. Un modelo sugerido es el Sistema de SARLAFT cuyo objetivo es la disminución de la probabilidad de pérdida, y ayuda $\mathrm{r}$ a la gestión de identificar, evaluar, medir, mitigar y monitorear. 


\section{REFERENCIAS}

Arias Moncada, R. (Julio de 2015). La Banca y la Prevención del Lavado de Activos en Ecuador (tesis de maestria). Facultad de Ciemcias Económicas. Universidad de Guayaquil. Obtenido de http://repositorio.ug.edu.ec/bitstream/redug/7797/1/LA\%20BANCA\%20Y\%20LA\%20PRE VENCI\%C3\%93N\%20DEL\%20LAVADO\%20DE\%20ACTIVOS\%20EN\%20EL\%20ECU ADOR.pdf

CICAD, C. I. (s.f.). Ecuador. Informe de Evaluación sobre Políticas de Drogas 2019. Organización de los Estados Americanos (OEA). Obtenido de http://www.cicad.oas.org/mem/reports/7/Full_Eval/Ecuador-7thRd-ESP.pdf

FGE. (Agosto de 2017). Modelo de Gestión Integral. Fiscalía General del Estado. Ecuador. Obtenido de https://www.fiscalia.gob.ec/pdf/Modelo-Gestion-FGE-2017-2023.pdf GAFILAT. (s.f.). ¿QUÉ ES EL GAFILAT? Recuperado el Diciembre de 2019, de https://www.gafilat.org/index.php/es/gafilat/que-es-gafilat

GAFISUD. (2011). Informe de evaluación mutua sobre lucha contra el lavado de activos y el financiamiento del terrorismo. . Ecuador.

Guerrero Luzuriaga, A. d., Marín Guamán, M. A., \& Bonilla Jurado, D. M. (2019). El lavado de activos y su influencia en la productividad y el y el desarrollo económico de un país. Revista Espacios, 40(18). Obtenido de https://www.revistaespacios.com/a19v40n18/a19v40n18p22.pdf

ICONTEC. (2004). Norma Técnica Colombia NTC 5254 Gestión del Riesgo. Obtenido de https://syeconsultoress.files.wordpress.com/2018/09/ntc-5254-gestion-del-riesgo.pdf

Infolaft. (s.f.). Infolaft. Obtenido de https://www.infolaft.com/sarlaft-los-sistemas-vigentescolombia-sector/

Infolaft. (s.f.). SAGRILAFT: ¿qué es y cómo implementarlo? Obtenido de Infolaft: https://www.infolaft.com/sagrlaft-todo-lo-que-debe-saber/

Mariño Becerra, G. Y., Chaparro Gómez, F. d., \& Medina Sandoval, I. (Julio-Diciembre de 2014). Determinantes en la prevención del riesgo para el lavado de activos y la financiación del terrorismo (LA/FT) en el sector real. AD-minister, 25, 7-35. Obtenido de http://www.scielo.org.co/pdf/adter/n25/n25a1.pdf

PGE. (s.f.). Recuperado el Diciembre de 2019, de Procuraduría General del Estado. República del Ecuador:

http://www.pge.gob.ec/?option=com_azurapagebuilder\&view=page\&id=165\#: :text=Duran te $202015 \% 2 \mathrm{C} \% 201 \mathrm{a} \% 20$ Procuradur\%C3\%ADa\%20General,de\%20que\%20su\%20imple mentaci\%C3\%B3n\%20es 


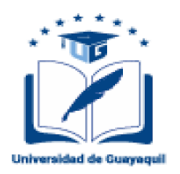

PGE. (Octubre de 2015). Ecuador recibió inofrme favorable para su exclusión definitiva de la clasificación GAFI. Procuraduría General del Estado. República del Ecuador. Obtenido de http://www.pge.gob.ec/index.php/2014-10-01-02-32-39/boletines2/item/173-ecuador- recibioinforme-favorable-para-su-exclusion-de-la-clasificacion-del-gafi

Saá, G. (Enero de 2013). La Normativa Ecuatoriana sobre el Lavado de Activos de las Instituciones del Sistema Financiero en el Ecuador: Propuesta de un Modelo de cumplimiento en el Banco Ecuatoriano de la Vivienda (tesis de maestría). Universidad Tecnológica Equinoccial.

Salazar Méndez, D. (2019). Informe de Gestión Anual Ejercicio 2018. Unidad de Anaálisis Financiero y Económico, (UAFE). Obtenido de https://www.uafe.gob.ec/wpcontent/uploads/downloads/2019/03/M-Informe-de-Gestion-2018-f.pdf

Salgado, W. (2010). Banca de Desarrollo en el Ecuador. N.U. CEPAL, Unidad de Estudios del Desarrollo. Agencia Española de Cooperación Internacional para el Desarrollo. Obtenido de https://repositorio.cepal.org/bitstream/handle/11362/5208/1/lcl3260.pdf

SCVS. (2019). Normas de Prevención de Lavado de Activos, Financiamiento del Terrorismo y otros Delitos. Superintendencia de Compañías, Valores y Seguros, Segundo Suplemento del Registro Oficial 96, 9-XII-2019. Obtenido de https://www.gob.ec/sites/default/files/regulations/202003/Documento_NORMAS- PREVENCI\%C3\%93N-LAVADO-ACTIVOS.pdf

SEPS. (2020). Superintendencia de la Economía Popular y Solidaria del Ecuador. Obtenido de https://www.seps.gob.ec/interna?-que-es-la-seps-

Superintencia de Bancos. (29 de Mayo de 2020). Normas de Control para las Entidades de los Sectores Financieros Público y Privado. Obtenido de uperbancos.gob.ec/bancos/wpcontent/uploads/downloads/2020/06/L1_IX_cap_VI.pdf

Superintendencia de la Economía Solidaria. (2017). Guía de Gestión del Riesgo de LA/FT para el Sector Solidario. Ministerio de Hacienda y Crédito Público. República de Colombia.

Obtenido

de

http://www.supersolidaria.gov.co/sites/default/files/public/SARLAFT/cartilla_guia_de_gesti on_del_riesgo00.pdf

UAFE. (2020). Informe de Gestión 2019. Unidad de Análisis Financiero y Económico. Obtenido de https://www.uafe.gob.ec/wp-content/uploads/downloads/2020/04/M-Informe-consolidado- 2019.pdf

UAFE. (2021). Ecuador se estrena como líder de uno de los grupos más importantes del mundo contra el lavado de activos. Boletín de Prensa UAFE-No.03-2021. Quito: Unidad de Análisis Financiero y Económico del Ecuador. Obtenido de https://www.uafe.gob.ec/ecuador-se-estrena-como-lider-deuno-de-los-grupos-mas- importantes-del-mundo-contra-el-lavado-de-activos/

UAFE. (2021). Informe de Gestión Enero-Diciembre 2020. Unidad de Análisis Financiero y Económico. Obtenido de https://www.uafe.gob.ec/wp- content/uploads/downloads/2021/06/M- 
INFORME-DE-GESTION-2020.pdf

UIAF. (2019). Guía de Normativad ALA/CFT. Unidad de Información y Análisis Financiero.

Obtenido

https://www.dian.gov.co/fizcalizacioncontrol/Documents/Guia\%20de\%20normatividad\%20

ALACFT.pdf

UNODC, O. d. (2017). Guía anticorrupción para las empresas. Basado en el Estatuto Anticorrupción. Bogotá: Oficina de las Naciones Unidas contra la Droga y el Delito , Cámara de Comercio de Bogotá, el Ministerio de Justicia, y el Derecho, la Embajada Británica y la alianza Negocios Responsables y Seguros(NSR).

Zabala Leal, T. D. (2019). Mitigación del Riesgo de Lavado de Activos y Financiación del Terrorismo. Editorial Politécnico Grancolombiano. Obtenido de https://www.researchgate.net/publication/342277876_MITIGACION_DEL_RIESGO_DE_ LAVADO_DE_ACTIVOS_Y_FINANCIACION_DEL_TERRORISMO 